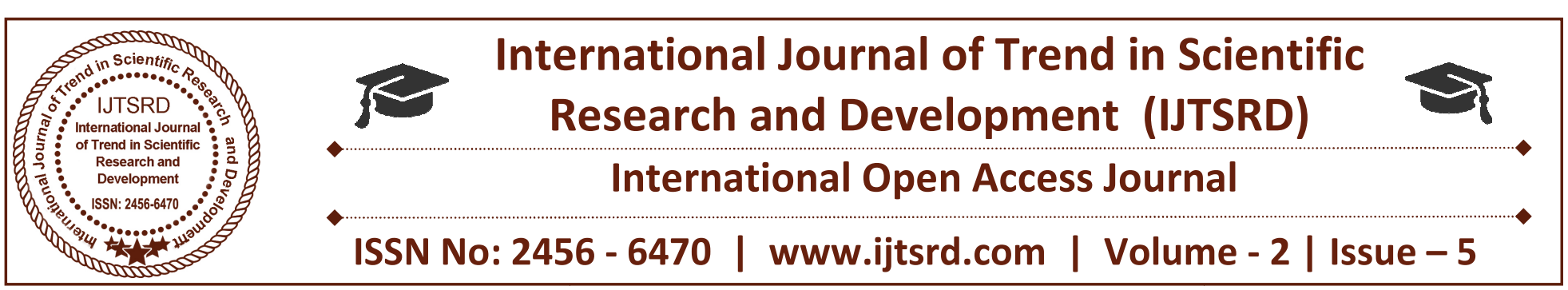

\title{
Turning Diffusion Based Image Colorization Into Efficient Color Compression
}

\author{
Syed Yunus ${ }^{1}$, Dr. Mohammed Abdul Waheed ${ }^{2}$ \\ ${ }^{1}$ Student, ${ }^{2}$ Professor \& Course Co-ordinator \\ Visvesvaraya Technological University Centre For PG Studies, Kalaburagi, Karnataka, India
}

\begin{abstract}
Neigh-boring pixels with comparable gray-values likewise have comparative hues. In the first place they change the picture to YUV space to acquire a arrangement shading decay. At that point they limit the distinction of every obscure shading pixel in YUV space to a biased normal of its $3 \times 3$ neighbor-hood.
\end{abstract}

Then this undertaking is tedious and dull, analysts go for limiting the measure of client communication that is required while as yet enabling craftsmen to impact the outcome. The technique for Levin et al is a traditional separate replica for knock oriented colorization there the client recommends a couple of shading writes and the calculation fills in the absent hues. In picture pressure, at first look a disconnected field, dispersion oriented codec's seek after comparable thoughts. There are likewise different non-neighborhood approaches for colorization: They have the favorable position that they can proliferate shading all around to all picture territories that have comparable structure.

Keywords: all picture territories that have comparable structure.

\section{INTRODUCTION}

Coloring the image is a for all intents and purposes significant picture handling errand that has its starting points in the film business. In the 1971s, numerous mono-chromatic movies were populer physically to incorporate shading. Sy-kora ET gives a decent diagram over the historical backdrop of premature colorization techniques. Then this undertaking is tedious and dull, analysts go for limiting the measure of client communication that is required while as yet enabling craftsmen to impact the outcome. The technique for Levin et al is a traditional separate replica for knock oriented colorization there the client recommends a couple of shading writes and the calculation fills in the absent hues. In picture pressure, at first look a disconnected field, dispersion oriented codec's seek after comparable thoughts.

They make a scanty portrayal of the picture via precisely choosing and putting away just a little part of pixels. The actually roused dissemination course engenders this known data into the absent regions to in paint (reproduce) the full picture in decompression. The R-EED codec of Schmaltz et al, joins the recreation capacities of edge-upgrading anisotropic dispersion with effective entropy coding to outflank the built up change based coders.

A na"1ve augmentation to shading pictures in a RG-B shading space likewise subsists. Be that as it may, it isn't aggressive to the JPE-G relatives, since it doesn't give the alternative to profit by the possessions of the person chart framework. Both JPE-G and JPEG-2000 misuse the way that the human observation esteems arrangement higher than shading. Specifically, they utilize shading spaces that disintegrate the picture into splendor and shading data to pack shading diverts in a coarser way

We build up a cozy relationship of our dissemination structure to crafted by Levin-et al,. They limit a separate vitality that depends on a straightforward suspicion: Neigh-boring pixels with comparable grayvalues likewise have comparative hues. In the first place they change the picture to YU-V space to acquire a arrangement shading decay. At that point they limit the distinction of every obscure shading 
pixel in YU-V space to a biased normal of its $3 \times 3$ neighbor-hood.

There are likewise different non-neighborhood approaches for colorization: They have the favorable position that they can proliferate shading all around to all picture territories that have comparable structure. For example, Yat-ziv and Sapiro normal known hues all inclusive as per power, while Luan assemble pixel's just nearby by force and all around by surface closeness.

The mapping amongst shine and shading esteems is gotten by Quang-et al, by methods for a mechanism knowledge methodology, and Yang-engenders shading data with a repeated reciprocal channel. For the particular class of mono-chromatic manga funnies, $\mathrm{Qu}$-al utilize grouping as per surface likeness. While non-nearby methodologies can yield better outcomes, they additionally have a tendency to accomplish this at the cost of calculation many-sided quality that can be a restricting component in time-basic function like video de-compression. That our pressure codec we depend on nearby, dissemination based colorization,

\section{Literature Survey}

Framework think about is an examination about existing framework. Here we will think about in insight about the current framework and its execution and proposed framework and its execution.

COLORIZATION is a for all intents and purposes pertinent picture preparing assignment that has its causes in the motion picture manufacturing. In the 1971s, numerous mono-chromatic movies were premastered physically to incorporate shading. Sykora et al, give a decent outline over the historical backdrop of soon colorization techniques. Since this errand is tedious and repetitive, analysts go for limiting the measure of client collaboration that is required while as yet enabling specialists to impact the outcome. The technique for Levin et al. [2] is an established separate model for knock oriented colorization where the client recommends a couple of shading jots and the calculation fill's in the absent hues. In picture pressure, at first look a random field, dissemination based codec's seek after comparative thoughts.

They make an inadequate portrayal of the picture via deliberately choosing and putting away just a little division of pixels. A physically enlivened dissemination process spreads this known data into the missing zones to in paint (remake) the full picture in decompression. The R-EED codec of Schmaltz et al, consolidates the recreation capacities of edgeupgrading anisotropic dispersion with proficient entropy coding to beat the set up change based.

\section{System Architecture}

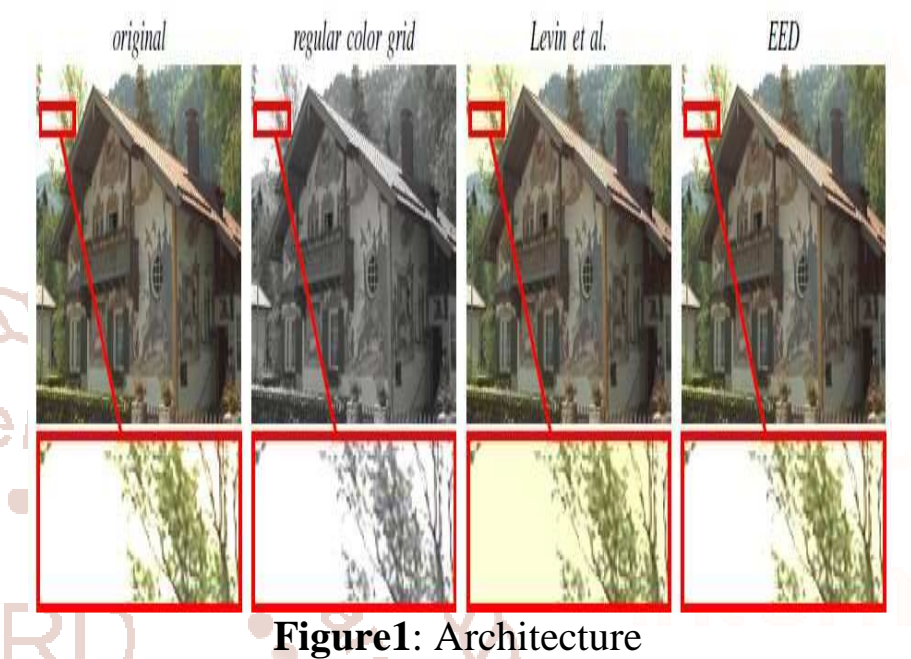

\section{Methodology}

In our paper, we set up a cozy relationship of our dispersion structure to crafted by Levin-et al. They limit a discrete vitality that depends on a straightforward suspicion: Neigh-boring pixels with comparable gray-values additionally have comparable hues. Initially they change the picture to YU-V space to get a structure-shading deterioration. At that point they limit the distinction of every obscure shading pixel in YU-V space to a weighted normal of its $3{ }_{-} 3$ neigh-borhood. In this work, we plan to deal with edge conservation specifically in a nonstop vitality definition.

This group coloring techniques as stroke-or illustration based. In stroke oriented techniques, the client physically determines a little measure of shading jots on a solitary picture or a grouping of pictures. Interestingly, case based calculations require one or numerous completely hued pictures that are like the gray scale picture to be colorized.

Given approach is helpful for video groupings and can be joined with stroke-based methodologies: A solitary edge can be shaded with scrawls and fill in for instance for the rest of the edges. Because of our attention on single pictures, we just consider the stroke-based approach. For a survey for coloring by case, we allude to Luan-all. 
The decipher coloring from an inclination area point of view and gives a system with edge-safeguarding requirements that incorporates coloring among different errands. Casa-ca et al. [13] accomplish sharp edges with a totally extraordinary advance: They first utilize the shading scrawls to parcel the picture into sections and after that spread shading as per portion marks.

Crafted by Levin promoted stroke based strategies that add shading to dark esteem pictures as indicated by a little measure of client determined shading tests, Despite the fact that such reproductions from inadequate information recommend a conceivable use in pressure, just couple of endeavors were made so far toward this path. Dispersion based pressure techniques seek after a comparative thought: They store just couple of picture pixels and in-paint the absent districts. Regardless of this nearby connection and an absence of dispersion based shading codec's, colorization thoughts were so far just coordinated into change based methodologies, for example, JPEG.

There are additionally different non-nearby methodologies for colorization: They have the preferred standpoint that they can engender shading all inclusive to all picture zones that have comparable structure. The example is a normal known hues all inclusive as indicated by power, while Luan et al. [9] aggregate pixels just locally by power and all inclusive by surface comparability.

\section{Results and Discussion}

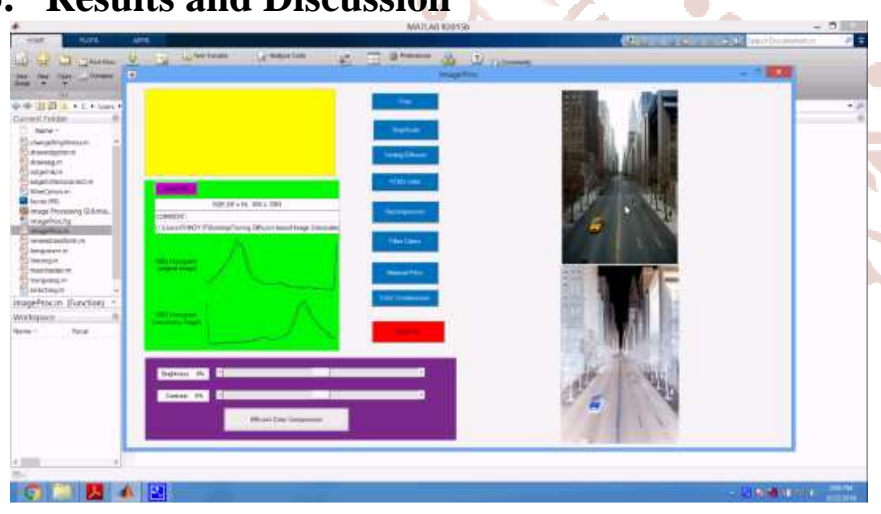

\section{Conclusion and Future Scope}

We have introduced another dissemination oriented coloring system in $\mathrm{YC}-\mathrm{bCr}$ space and have demonstrated that the technique for Levin-et al. is identified with isotropic dispersion in-painting. Our luma's -guided anisotropic dispersion outflanks the approach of Levin's. What's more, can recreate shading from exceptionally scanty known information. That makes it a significant apparatus for pressure: Our luma inclination mode acquaints keen coding with PDE-orinted pressure. This gives another vital advance towards achieving an indistinguishable complex level of building from change oriented codec's. An assessment on the Kodak database exhibits that on pictures with medium measures of surface, L-P scheme can beat both PD-E-and change based contenders. Notwithstanding for exceptionally finished pictures, our strategy beats JPE-G and approaches the nature of JPEG-2000.

The future implementation may include the real time image colorization scheme, which can be applied directly to the video files only. This will make the process very convenient and also one can embed this scheme to be added to the camera module, which will set the colorization scheme to work at the time of taking the picture itself.

\section{Acknowledgment}

The authors would like to thank a great support.

\section{References}

1. D. Sykora "Unsupervised colorization of dark ' and-white kid's shows 2004

2. A. Levin, "Colorization utilizing enhancement 2004.

3. C. Schmaltz, "Understanding, upgrading, and expanding information pressure with anisotropic dissemination," International Journal of Computer Vision 2014.

4. J-Weickert, "Hypothetical establishments of anisotropic dispersion in picture handling," Computing Supplement 1996.

5. W. B. Penne baker: Still Image Data Compression Standard. New York: Springer, 1992.

6. D. S. Taubman: Image Compression Fundamentals, Standards and Practice. Boston: Kluwer, 2002.

7. P. Subside "Shading picture pressure with anisotropic dissemination 2014

8. Eastman Kodak Company Accessible

9. Q. Luan, F "Characteristic picture colorization," in Proc. eighteenth Euro graphics Conference on Rendering Techniques.

10. Y. - C. Huang" A versatile edge location based colorization calculation and its applications," in Proc. thirteenth Annual ACM International Conference on Multimedia, Singapore, Nov. 2005. 